Volume 135, Number 11, November 2007, Pages 3477-3483

S 0002-9939(07)08964-2

Article electronically published on June 29, 2007

\title{
AMPLENESS OF INTERSECTIONS OF TRANSLATES OF THETA DIVISORS IN AN ABELIAN FOURFOLD
}

\author{
O. DEBARRE AND E. IZADI
}

(Communicated by Michael Stillman)

\begin{abstract}
We prove the ampleness of the cotangent bundle of the intersection of two general translates of a theta divosor of the Jacobian of a general curve of genus 4. From this, we deduce the same result in a general, principally polarized abelian variety of dimension 4 .
\end{abstract}

\section{INTRODUCTION}

Varieties with ample cotangent bundle are interesting from many points of view. If $X$ is such a variety, defined over a field $\mathbf{k}$, then

- (geometric) all subvarieties of $X$ are of general type and there are only finitely many rational maps from any fixed projective variety to $X([\mathrm{NS}])$;

- (analytic) if $\mathbf{k}=\mathbf{C}$, any holomorphic map $\mathbf{C} \rightarrow X$ is constant ([De], (3.1));

- (arithmetic) if $\mathbf{k}$ is a number field, the set of $\mathbf{k}$-rational points of $X$ is conjectured to be finite (see $[\mathrm{Mo}]$; the analogous statement over function fields of curves is known to hold by $[\mathrm{N}]$ or $[\mathrm{MD}]$ ).

However, there are relatively few concrete examples of such varieties. Bogomolov was the first to give a general procedure to produce such examples. His construction is explained in [D], and in that article, more examples are constructed: it is shown that given any principally polarized abelian variety $(A, \Theta)$, an integer $n \geq \frac{1}{2} \operatorname{dim} A$, and sufficiently ample (i.e., algebraically equivalent to sufficiently high multiples of $\Theta$ ) general divisors $D_{1}, \ldots, D_{n}$, the smooth variety $D_{1} \cap \cdots \cap D_{n}$ has ample cotangent bundle. In this paper we prove an analogous result for general abelian fourfolds. We work over an algebraically closed field $\mathbf{k}$.

Theorem 1. Let $(A, \Theta)$ be a general principally polarized abelian fourfold. For $a \in A$ general, the smooth surface $\Theta \cap \Theta_{a}$ has ample cotangent bundle.

Here $\Theta_{a}$ denotes the translate $\Theta+a$ of $\Theta$ by $a$. Our proof shows that the conclusion of the theorem already holds on a general Jacobian fourfold.

Received by the editors June 23, 2005 and, in revised form, August 21, 2006.

2000 Mathematics Subject Classification. Primary 14K12; Secondary 14M10, 14F10.

Key words and phrases. Ample cotangent bundle, abelian variety, algebraic surface, complete intersection.

This work was done while O. Debarre was visiting the Department of Mathematics of the University of Georgia, at the invitation of E. Izadi. He is grateful to E. Izadi for her hospitality. Both authors are grateful to the University of Georgia for its support. 


\section{The ampleness of $\Omega_{\Theta \cap \Theta_{a}}$}

Let $(A, \Theta)$ be a principally polarized abelian fourfold. Assume $\Theta \cap \Theta_{a}$ is a smooth surface. The cotangent bundle $\Omega_{\Theta \cap \Theta_{a}}$ fits into the exact sequence of conormal and cotangent bundles

$$
\left.0 \longrightarrow \mathscr{O}_{\Theta \cap \Theta_{a}}(-\Theta) \oplus \mathscr{O}_{\Theta \cap \Theta_{a}}\left(-\Theta_{a}\right) \longrightarrow \Omega_{A}\right|_{\Theta \cap \Theta_{a}} \longrightarrow \Omega_{\Theta \cap \Theta_{a}} \longrightarrow 0
$$

Being a quotient of a trivial vector bundle, it is generated by its global sections, which are identified with $H^{0}\left(\Theta \cap \Theta_{a},\left.\Omega_{A}\right|_{\Theta \cap \Theta_{a}}\right) \simeq H^{0}\left(A, \Omega_{A}\right)$. To show that $\Omega_{\Theta \cap \Theta_{a}}$ is ample, we must show that the associated morphism

$$
\psi_{a}: \mathbf{P}\left(\Omega_{\Theta \cap \Theta_{a}}\right) \longrightarrow \mathbf{P}\left(H^{0}\left(A, \Omega_{A}\right)\right)
$$

is finite (we follow Grothendieck's notation: given a coherent sheaf $\mathscr{E}$ on a scheme, $\mathbf{P}(\mathscr{E})=\operatorname{Proj}(\operatorname{Sym} \mathscr{E}))$. More concretely, a point in $\mathbf{P}\left(H^{0}\left(A, \Omega_{A}\right)\right)$ corresponds to a hyperplane in $H^{0}\left(A, \Omega_{A}\right)$, or to a line $\ell$ in $T_{A, 0}$, and

$$
\mathbf{P}\left(\Omega_{\Theta \cap \Theta_{a}}\right)=\left\{(\ell, x) \in \mathbf{P}\left(H^{0}\left(A, \Omega_{A}\right)\right) \times\left(\Theta \cap \Theta_{a}\right) \mid \ell \subset T_{\Theta \cap \Theta_{a}, x}\right\},
$$

where $T_{A, 0}$ and $T_{A, x}$ are identified by translation by $x$, and $\psi_{a}$ is the first projection.

In other words, to prove that $\Omega_{\Theta \cap \Theta_{a}}$ is ample, we must prove that, for any nonzero $\partial^{\prime} \in T_{A, 0}$, the set of points $x \in \Theta \cap \Theta_{a}$ such that $\partial^{\prime} \in T_{\Theta \cap \Theta_{a}, x}$ is finite. We denote by $\left[\partial^{\prime}\right]$ the point of $\mathbf{P}\left(H^{0}\left(A, \Omega_{A}\right)\right)$ determined by $\partial^{\prime}$.

\section{THE DIVISOR $\Theta \cap \partial \Theta$}

Let $(A, \Theta)$ be a principally polarized abelian variety and let $\theta$ be a nonzero section of $\mathscr{O}_{A}(\Theta)$. We define, for any $\partial$ in $T_{A, 0}$, a section $\partial \theta$ of $\mathscr{O}_{\Theta}(\Theta)$ by the requirement that for any open set $U$ of $A$ and any trivialization $\varphi:\left.\mathscr{O}_{U} \stackrel{\sim}{\longrightarrow} \mathscr{O}_{\Theta}(\Theta)\right|_{U}$, we have $\partial \theta=\left.\varphi\left(\partial\left(\varphi^{-1}(\theta)\right)\right)\right|_{\Theta}$ in $U \cap \Theta$. We denote its zero locus by $\Theta \cap \partial \Theta$. Settheoretically, $\Theta \cap \partial \Theta$ is the set of points $x$ of $\Theta$ where the Zariski tangent space $T_{\Theta, x}$ contains $\partial$.

The differential of the isomorphism $A \rightarrow \operatorname{Pic}^{0}(A)$ induced by the polarization $\Theta$ identifies $T_{A, 0}$ with $T_{\mathrm{Pic}^{0}(A), 0} \simeq H^{1}\left(A, \mathscr{O}_{A}\right)$. The exact sequence

$$
0 \longrightarrow \mathscr{O}_{A} \longrightarrow \mathscr{O}_{A}(\Theta) \longrightarrow \mathscr{O}_{\Theta}(\Theta) \longrightarrow 0
$$

yields a composed isomorphism

$$
H^{0}\left(\Theta, \mathscr{O}_{\Theta}(\Theta)\right) \stackrel{\sim}{\longrightarrow} H^{1}\left(A, \mathscr{O}_{A}\right) \stackrel{\sim}{\longrightarrow} T_{A, 0}
$$

whose inverse is given by

$$
\partial \longmapsto \partial \theta \text {. }
$$

When $A$ has dimension 4, the ampleness of the cotangent bundle of $\Theta \cap \Theta_{a}$ is equivalent to the following: for all nonzero $\partial^{\prime} \in T_{A, 0}$, the scheme $\Theta \cap \partial^{\prime} \Theta \cap \Theta_{a} \cap \partial^{\prime} \Theta_{a}$ is finite.

For $\partial \neq 0$, the scheme $\Theta \cap \partial \Theta$ is a limit of intersections of translates of $\Theta$ in the following sense. Let $m: \Theta \times A \rightarrow A$ be the morphism $(x, y) \mapsto x-y$ and let $\mathscr{T}=m^{-1}(\Theta)$. The first projection $\Theta \times A \rightarrow \Theta$ identifies $\Theta \cap \Theta_{a}$ with the fiber at $a$ of the second projection

$$
p: \mathscr{T} \longrightarrow A
$$

If $\widetilde{A} \rightarrow A$ is the blowup of 0 , with exceptional divisor $\mathbf{P}\left(\Omega_{A, 0}\right)$, and $\widetilde{\mathscr{T}}$ is the strict transform of $\mathscr{T}$ in $\Theta \times \widetilde{A} \rightarrow \Theta \times A$, we obtain a family

$$
\tilde{p}: \widetilde{\mathscr{T}} \longrightarrow \widetilde{A}
$$


whose fiber at $[\partial] \in \mathbf{P}\left(\Omega_{A, 0}\right)$ is isomorphic to $\Theta \cap \partial \Theta$. If $\Theta$ is irreducible, this is a flat family of subschemes of codimension 2 of $A$.

We will study the ampleness of the cotangent bundle of $\Theta \cap \Theta_{a}$ by letting it specialize to $\Theta \cap \partial \Theta$. More precisely, if we consider

$$
\mathbf{P}=\left\{(\ell, x, \tilde{a}) \in \mathbf{P}\left(H^{0}\left(A, \Omega_{A}\right)\right) \times \widetilde{\mathscr{T}} \mid \ell \subset T_{\tilde{p}^{-1}(\tilde{a}),(x, \tilde{a})}\right\},
$$

the projection $\psi: \mathbf{P} \rightarrow \mathbf{P}\left(H^{0}\left(A, \Omega_{A}\right)\right) \times \widetilde{A}$ restricts to $\psi_{a}$ over $\mathbf{P}\left(H^{0}\left(A, \Omega_{A}\right)\right) \times\{a\}$, for $a \in A$ nonzero, and to a morphism

$$
\psi_{\partial}: \mathbf{P}_{\partial}=\left\{(\ell, x) \in \mathbf{P}\left(H^{0}\left(A, \Omega_{A}\right)\right) \times(\Theta \cap \partial \Theta) \mid \ell \subset T_{\Theta \cap \partial \Theta, x}\right\} \rightarrow \mathbf{P}\left(H^{0}\left(A, \Omega_{A}\right)\right)
$$

over $\mathbf{P}\left(H^{0}\left(A, \Omega_{A}\right)\right) \times\{[\partial]\}$, for $\partial \in T_{A, 0}$ nonzero. If $\psi_{\partial}$ is finite, the same will be true for $\psi_{a}$ for general $a$ in $A$.

\section{The finiteness of $\Theta \cap \partial \Theta \cap \partial^{\prime} \Theta \cap \partial \partial^{\prime} \Theta$}

Again let $(A, \Theta)$ be a principally polarized abelian fourfold. As explained above, we would like to find a nonzero element $\partial$ of $T_{A, 0}$ such that the morphism

$$
\psi_{\partial}: \mathbf{P}_{\partial} \longrightarrow \mathbf{P}\left(H^{0}\left(A, \Omega_{A}\right)\right)
$$

is finite. If $\partial^{\prime}$ is a nonzero element of $T_{A, 0}$, we may define as above a section $\partial \partial^{\prime} \theta$ of $\mathscr{O}_{\Theta \cap \partial \Theta \cap \partial^{\prime} \Theta}(\Theta)$ whose zero locus we denote by $\Theta \cap \partial \Theta \cap \partial^{\prime} \Theta \cap \partial \partial^{\prime} \Theta$ and which is isomorphic to the fiber $\psi_{\partial}^{-1}\left(\left[\partial^{\prime}\right]\right)$.

Unfortunately, this scheme has codimension at most 3 for $\partial^{\prime}=\partial$. We will first prove that for $A$ a general Jacobian and $\partial$ general in $T_{A, 0}$, this is the only positive-dimensional fiber of $\psi_{\partial}$.

Let $C$ be a smooth curve of genus 4 , take $A=\operatorname{Pic}^{3} C$, and let $\Theta \subset A$ be Riemann's theta divisor parametrizing effective divisors of degree 3 on $C$.

Proposition 2. For $C$ and $\partial$ general, all fibers of the morphism $\psi_{\partial}$ are finite, except for that of $[\partial]$.

Proof. Take $\partial \in T_{A, 0}$ nonzero and let $S_{\partial}$ be the (local complete intersection) surface $\Theta \cap \partial \Theta$. Noting that the restriction $H^{1}\left(A, \mathscr{O}_{A}\right) \rightarrow H^{1}\left(\Theta, \mathscr{O}_{\Theta}\right)$ is bijective and using the isomorphism (2), we obtain from the exact sequence

$$
0 \longrightarrow \mathscr{O}_{\Theta} \stackrel{\partial \theta}{\longrightarrow} \mathscr{O}_{\Theta}(\Theta) \longrightarrow \mathscr{O}_{S_{\partial}}(\Theta) \longrightarrow 0
$$

an exact sequence

$$
0 \longrightarrow \mathbf{k} \stackrel{\cdot \partial}{\longrightarrow} \begin{array}{ccc}
T_{A, 0} & \longrightarrow & H^{0}\left(S_{\partial}, \mathscr{O}_{S_{\partial}}(\Theta)\right) \\
\partial^{\prime} & \longmapsto & \partial^{\prime} \theta
\end{array}
$$

Let $\partial^{\prime} \in T_{A, 0}$ be nonzero. If $\Gamma=\Theta \cap \partial \Theta \cap \partial^{\prime} \Theta$ is an integral, i.e., irreducible and reduced, curve, $\partial^{\prime} \theta$ is not a zero divisor in $\mathscr{O}_{S_{\partial}}$ and again, since $H^{1}\left(A, \mathscr{O}_{A}\right) \rightarrow$ $H^{1}\left(S_{\partial}, \mathscr{O}_{S_{\partial}}\right)$ is bijective, we obtain from the exact sequence

$$
0 \longrightarrow \mathscr{O}_{S_{\partial}} \stackrel{\partial^{\prime} \theta}{\longrightarrow} \mathscr{O}_{S_{\partial}}(\Theta) \longrightarrow \mathscr{O}_{\Gamma}(\Theta) \longrightarrow 0
$$

a coboundary map $H^{0}\left(\Gamma, \mathscr{O}_{\Gamma}(\Theta)\right) \rightarrow T_{A, 0}$ that sends $\partial \partial^{\prime} \theta$ to $\partial$. This section is, in particular, nonzero; hence its zero locus $\Theta \cap \partial \Theta \cap \partial^{\prime} \Theta \cap \partial \partial^{\prime} \Theta$ is finite, which is what we need to prove.

We assume from now on that $C$ is not hyperelliptic and we identify it with its canonical model in $\mathbf{P}^{3}=\mathbf{P}\left(H^{0}\left(C, \omega_{C}\right)\right)=\mathbf{P}\left(H^{0}\left(A, \Omega_{A}\right)\right)$, where it is the intersection of a quadric $Q$ (which will be assumed to be smooth) and a cubic. 
The projectivization of the tangent space to $\Theta$ at a point corresponding to a divisor $D$ of degree 3 such that $h^{0}(C, D)=1$ is the plane spanned in $\mathbf{P}^{3}$ by the points of $D$. The underlying reduced curve $\Gamma_{\text {red }}$ therefore parametrizes effective divisors of degree 3 on $C$ that lie in a plane that contains the line $\ell_{\partial, \partial^{\prime}}$ spanned by $[\partial]$ and $\left[\partial^{\prime}\right]$. We will distinguish several cases depending on the relative positions of $[\partial],\left[\partial^{\prime}\right]$, and $C$ in $\mathbf{P}^{3}$.

We first introduce some notation, following [I]: given a pencil $g_{e}^{1}$ on $C$ with reduced base locus, we define, for any $d \in\{1, \ldots, e\}$, a reduced curve in the $d$-th symmetric power $C^{(d)}$ by setting

$$
X_{d}\left(g_{e}^{1}\right)=\left\{p_{1}+\cdots+p_{d} \in C^{(d)} \mid \exists D \in C^{(e-d)}, D+p_{1}+\cdots+p_{d} \in g_{e}^{1}\right\} .
$$

3.1. Case 1: $\ell_{\partial, \partial^{\prime}} \cap C=\varnothing$. The planes containing $\ell_{\partial, \partial^{\prime}}$ cut on $C$ the divisors of a base-point-free $g_{6}^{1}$ contained in $\left|\omega_{C}\right|$, and the curve $\Gamma_{\text {red }}$ is the image in $\Theta$ of the curve $X_{3}\left(g_{6}^{1}\right) \subset C^{(3)}$. It follows from [ACGH], Lemma VIII.(3.2) that the cohomology class of $\Gamma_{\text {red }}$ is $[\Theta]^{3}$, so $\Gamma$ is reduced.

The associated map $\phi: C \rightarrow\left(g_{6}^{1}\right)^{*}=\mathbf{P}^{1}$ coincides with the projection of $C \subset \mathbf{P}^{3}$ from the line $\ell_{\partial, \partial^{\prime}}$. The lemma below shows that the monodromy group $G$ of $\phi$ is the full symmetric group $\mathfrak{S}_{6}$. This implies that $\Gamma$ is integral, and we are done in this case.

Lemma 3. For $C$ general and $[\partial] \notin Q$, the group $G$ is 2-transitive and contains a simple transposition.

Proof. The 2-transitivity of $G$ is equivalent to the irreducibility of the curve $X_{2}\left(g_{6}^{1}\right)$ in $C^{(2)}$.

Let $\pi: C^{2} \rightarrow C^{(2)}$ be the quotient map. For any divisor (resp. divisor class) $D$ on $C$, let $C_{D}$ be the unique divisor (resp. divisor class) on $C^{(2)}$ such that $\pi^{*} C_{D}=$ $p_{1}^{*} D+p_{2}^{*} D$. Let $\delta$ be the unique divisor class on $C^{(2)}$ such that $\pi^{*} \delta$ is linearly equivalent to the diagonal of $C^{2}$. We have the linear equivalence $X_{2}\left(g_{6}^{1}\right) \equiv C_{g_{6}^{1}}-\delta$ ([I], Lemma 2.1). Moreover, $\delta^{2}=-3$ and $\delta \cdot C_{D}=\operatorname{deg}(D)$.

Assume $C$ is sufficiently general so that the map

$$
\begin{aligned}
& \operatorname{Pic}(C) \oplus \mathbf{Z} \longrightarrow \operatorname{Pic}\left(C^{(2)}\right) \\
& (D, b) \quad \longmapsto \quad C_{D}-b \delta
\end{aligned}
$$

is bijective. If $X_{2}\left(g_{6}^{1}\right)$ is reducible, write the divisor class of a nontrivial union of components, say $Y$, as $C_{D}-b \delta$, so that the class of $X_{2}\left(g_{6}^{1}\right)-Y$ is $C_{g_{6}^{1}-D}-(1-b) \delta$. Replacing $Y$ with $X_{2}\left(g_{6}^{1}\right)-Y$ if necessary, we may assume $b \geq 0$.

We now use [I], Appendix 6.1: for any divisor $E$ on $C$, we have

$$
H^{0}\left(C^{(2)}, C_{E}\right) \simeq \operatorname{Sym}^{2} H^{0}(C, E) \text { and } H^{0}\left(C^{(2)}, C_{E}-\delta\right) \simeq \bigwedge^{2} H^{0}(C, E) .
$$

It follows that if $E$ is effective and $h^{0}(C, E)=1$, the linear system $\left|C_{E}-\delta\right|$ is empty, and $\left|C_{E}\right|=\left\{C_{E}\right\}$. Since our $g_{6}^{1}$ has no base points, $X_{2}\left(g_{6}^{1}\right)$ contains no such curve. It follows that $D$ moves in a pencil; hence $\operatorname{deg}(D) \geq 3$ since $C$ is not hyperelliptic. Since the diagonal is not a component of $X_{2}\left(g_{6}^{1}\right)$, we must have $\left(C_{g_{6}^{1}-D}-(1-b) \delta\right) \cdot \delta \geq 0$; hence $3 b \leq 9-\operatorname{deg}(D)$.

If $\operatorname{deg}(D) \geq 4$, we get $b \leq 1$, but this is absurd since $\left|C_{g_{6}^{1}-D}-(1-b) \delta\right|$ is then empty. Hence $D$ is a $g_{3}^{1}$ and $b \leq 2$. By [I], Appendix 6.3, the vector subspace $H^{0}\left(C^{(2)}, C_{g_{3}^{1}}-2 \delta\right) \subset H^{0}\left(C^{(2)}, C_{g_{3}^{1}}\right)$ is isomorphic to the space of quadratic forms 
that vanish on the image of $C \rightarrow\left(g_{3}^{1}\right)^{*}$, hence vanishes. We get $b \in\{0,1\}$ and, replacing $Y$ with $X_{2}\left(g_{6}^{1}\right)-Y$ if necessary, $Y \equiv C_{g_{3}^{1}}-\delta$. More precisely, $Y=X_{2}\left(g_{3}^{1}\right)$. The $g_{3}^{1}$ is given by one of the rulings of the quadric $Q$; hence $X_{2}\left(g_{3}^{1}\right)$ may be contained in $X_{2}\left(g_{6}^{1}\right)$ only if the line $\ell_{\partial, \partial^{\prime}}$ meets all lines of this ruling. Since $[\partial]$ is not in $Q$, this cannot happen and $X_{2}\left(g_{6}^{1}\right)$ is irreducible.

To prove that $G$ contains a simple transposition, we check that for $C$ general, there is a point $p \in C$ such that $\phi: C \rightarrow \mathbf{P}^{1}$ ramifies simply at $p$ and $p$ is the only ramification point of $\phi$ in its fiber.

The degree of the ramification locus is $6+6 \cdot 2=18$. If all the ramification points are either nonsimple or their fiber contains other ramification points, the support of the branch locus of $\phi$ in $\mathbf{P}^{1}$ contains at most 9 points. Such 6 -fold covers of $\mathbf{P}^{1}$ depend on at most $9-3=6$ parameters. Therefore, for a sufficiently general choice of $C$, the map $\phi$ will have at least 3 ramification points with the desired property. $C$.

We assume from now on that $[\partial]$ lies on no trisecants $([\partial] \notin Q)$ or tangents to

3.2. Case 2: $\ell_{\partial, \partial^{\prime}} \cap C=\{p\}$. Here we mean that the line $\ell_{\partial, \partial^{\prime}}$ is not tangent to $C$. In this case we have

$$
\Gamma_{\text {red }}=X_{3}\left(g_{5}^{1}\right) \cup\left(X_{2}\left(g_{5}^{1}\right)+p\right) \subset C^{(3)},
$$

where $g_{5}^{1} \subset\left|\omega_{C}\right|$ is the base-point-free pencil cut on $C$ by planes through $\ell_{\partial, \partial^{\prime}}$. As before, we see that $\Gamma$ is reduced. The involution

$$
\tau: x+y+z \longmapsto K_{C}-x-y-z
$$

exchanges $X_{3}\left(g_{5}^{1}\right)$ and $X_{2}\left(g_{5}^{1}\right)+p$. A similar (simpler) calculation as before shows that $X_{2}\left(g_{5}^{1}\right)$, hence also $X_{3}\left(g_{5}^{1}\right)$, is irreducible. As the scheme $\Gamma \cap \partial \partial^{\prime} \Theta$ is invariant under $\tau$, we see that if it contains one component of $\Gamma$, it also contains the other. This is therefore not possible; hence this scheme is finite.

3.3. Case 3: $\ell_{\partial, \partial^{\prime}} \cap C=\{p, q\}$. Here we mean that the line $\ell_{\partial, \partial^{\prime}}$ intersects $C$ in exactly two distinct points $p$ and $q$.

Let $\partial_{p}$ and $\partial_{q}$ be elements of $T_{A, 0}$ mapping to $p$ and $q$ respectively. Let $W_{p}$ be the image in $\Theta$ of $p+C^{(2)} \subset C^{(3)}$. We have

$$
\Theta \cap \partial_{p} \Theta=W_{p} \cup\left(K_{C}-W_{p}\right)=W_{p} \cup \tau\left(W_{p}\right) .
$$

Since $[\partial] \notin Q$, the linear system $\left|K_{C}-p-q\right|$ is a base-point-free $g_{4}^{1}$ and the curve $X_{2}\left(K_{C}-p-q\right)$ is irreducible as before. We have $\Gamma=\Theta \cap \partial \Theta \cap \partial^{\prime} \Theta=\Theta \cap \partial_{p} \Theta \cap \partial_{q} \Theta$ and we check that this curve is reduced and has four irreducible components:

$$
\begin{aligned}
\Gamma_{1} & =p+q+C, & \Gamma_{2} & =p+X_{2}\left(K_{C}-p-q\right), \\
\tau\left(\Gamma_{1}\right) & =X_{3}\left(K_{C}-p-q\right), & \tau\left(\Gamma_{2}\right) & =q+X_{2}\left(K_{C}-p-q\right) .
\end{aligned}
$$

If $\Theta \cap \partial \Theta \cap \partial^{\prime} \Theta \cap \partial \partial^{\prime} \Theta$ contains a component of $\Gamma$, it also contains its image by $\tau$. It will therefore be enough for our purpose to show that the section $\partial \partial^{\prime} \theta$ of $\mathscr{O}_{\Gamma}(\Theta)$ vanishes identically neither on $\Gamma_{1}$, nor on $\Gamma_{2}$ (both contained in $W_{p}$ ).

Let $\iota_{p+q}$ be the embedding $x \mapsto p+q+x$ of $C$ into $A$, with image $\Gamma_{1}$. We have $\iota_{p+q}^{*} \Theta \equiv K_{C}-p-q$. Let $p+p_{1}+p_{2}+p_{3}$ and $q+q_{1}+q_{2}+q_{3}$ be the divisors of $|K-p-q|$ containing $p$ and $q$. For a sufficiently general choice of $\partial$, these two divisors will be reduced and disjoint. 
Lemma 4. The section $\iota_{p+q}^{*} \partial_{p} \partial_{q} \theta$ vanishes identically and

$$
\begin{aligned}
& \operatorname{div}\left(\iota_{p+q}^{*} \partial_{p}^{2} \theta\right)=p+p_{1}+p_{2}+p_{3}, \\
& \operatorname{div}\left(\iota_{p+q}^{*} \partial_{q}^{2} \theta\right)=q+q_{1}+q_{2}+q_{3} .
\end{aligned}
$$

Proof. Let $\lambda \in \mathbf{k}$ and set $\partial_{\lambda}=\lambda \partial_{p}+\partial_{q}$. The support of

$$
\operatorname{div}\left(\partial_{p} \partial_{\lambda} \theta\right)=\Theta \cap \partial_{p} \Theta \cap \partial_{q} \Theta \cap \partial_{p} \partial_{\lambda} \Theta=\Theta \cap \partial_{p} \Theta \cap \partial_{\lambda} \Theta \cap \partial_{p} \partial_{\lambda} \Theta
$$

is the set of points of $\Theta \cap \partial_{p} \Theta=W_{p} \cup\left(K_{C}-W_{p}\right)$ whose tangent space contains $\partial_{\lambda}$.

It contains $p+q+x$ if the line $\langle q, x\rangle$ contains $\left[\partial_{\lambda}\right]$. In particular, $\partial_{p} \partial_{q} \theta$ vanishes identically on $p+q+C$ and $\partial_{p} \partial_{\lambda} \theta(2 p+q)=0$ for all $\lambda$. This implies $\partial_{p}^{2} \theta(2 p+q)=0$. Moreover, $\partial_{p} \partial_{\lambda} \theta(p+2 q) \neq 0$ if $\lambda \neq 0$. In particular, $\iota_{p+q}^{*} \partial_{p}^{2} \theta$ is a nonzero section of $\mathscr{O}_{C}\left(K_{C}-p-q\right)$ that vanishes at $p$, hence the lemma.

Write $\partial=\lambda \partial_{p}+\mu \partial_{q}$ and $\partial^{\prime}=\lambda^{\prime} \partial_{p}+\mu^{\prime} \partial_{q}$, so that

$$
\partial \partial^{\prime} \theta=\lambda \lambda^{\prime} \partial_{p}^{2} \theta+\left(\lambda \mu^{\prime}+\lambda^{\prime} \mu\right) \partial_{p} \partial_{q} \theta+\mu \mu^{\prime} \partial_{q}^{2} \theta .
$$

Since $[\partial]$ is not on $C$, both $\lambda$ and $\mu$ are not zero; hence $\partial \partial^{\prime} \theta$ does not vanish identically on $\Gamma_{1}$. We have

$$
\begin{aligned}
\Gamma_{1} \cap \Gamma_{2} & =\left\{p+q+q_{1}, p+q+q_{2}, p+q+q_{3}\right\}, \\
\Gamma_{1} \cap \tau\left(\Gamma_{2}\right) & =\left\{p+q+p_{1}, p+q+p_{2}, p+q+p_{3}\right\}, \\
\tau\left(\Gamma_{1}\right) \cap \Gamma_{2} & =\left\{\tau\left(p+q+p_{1}\right), \tau\left(p+q+p_{2}\right), \tau\left(p+q+p_{3}\right)\right\} .
\end{aligned}
$$

The section $\partial_{p} \partial_{q} \theta$ does not vanish identically on $\Gamma$, hence does not vanish identically on $\Gamma_{2}$. At $p+q+q_{1}$, both $\partial_{p} \partial_{q} \theta$ and $\partial_{q}^{2} \theta$ vanish, but $\partial_{p}^{2} \theta$ does not. At $\tau(p+q+$ $\left.p_{1}\right)$, both $\partial_{p} \partial_{q} \theta$ and $\partial_{p}^{2} \theta$ vanish, but $\partial_{q}^{2} \theta$ does not. It follows that the sections $\left.\partial_{p}^{2} \theta\right|_{\Gamma_{2}},\left.\partial_{p} \partial_{q} \theta\right|_{\Gamma_{2}}$, and $\left.\partial_{q}^{2} \theta\right|_{\Gamma_{2}}$ are linearly independent; hence $\partial \partial^{\prime} \theta$ does not vanish identically on $\Gamma_{2}$.

We have proved that in all cases, the zero set of $\partial \partial^{\prime} \theta$ on $\Gamma$ is finite. This completes the proof of Proposition 2.

\section{THE SCHEME $\Theta \cap \partial \Theta \cap \partial^{2} \Theta$}

The fiber of $\psi_{\partial}^{-1}([\partial])$ is one-dimensional, equal to $\Theta \cap \partial \Theta \cap \partial^{2} \Theta$. We now study this curve. Let $p$ be a general point of $C$. As above, we see that $\Theta \cap \partial_{p} \Theta \cap \partial_{p}^{2} \Theta$ has three irreducible components whose reduced underlying curves are

$$
\begin{gathered}
\Gamma_{1}=2 p+C, \quad \tau\left(\Gamma_{1}\right)=X_{3}\left(K_{C}-2 p\right), \\
\Gamma_{2}=\tau\left(\Gamma_{2}\right)=p+X_{2}\left(K_{C}-2 p\right),
\end{gathered}
$$

and $\Gamma_{1}$ and $\tau\left(\Gamma_{1}\right)$ have multiplicity 1 , whereas $\Gamma_{2}$ has multiplicity 2 .

Lemma 5. For $\partial$ general, $\Theta \cap \partial \Theta \cap \partial^{2} \Theta$ contains no translates of $C$.

Proof. A translate of $C$ is contained in $\Theta$ if and only if it is of the type $x+y+C$, with $x, y \in C$. It is contained in $\Theta \cap \partial \Theta$ if and only if for every $t \in C$, the plane $\langle x, y, t\rangle$ contains $[\partial]$. This is only possible if $[\partial]$ is on the line $\langle x, y\rangle$. For $\partial$ general, there are exactly six distinct secants to $C$ that contain $[\partial]$, none of which is trisecant or tangent. So there are exactly six distinct translates, say $x_{i}+y_{i}+C$, for $i \in\{1, \ldots, 6\}$, contained in $\Theta \cap \partial \Theta$. Since the set of secants to $C$ is irreducible, 
if one of these translates is contained in $\Theta \cap \partial \Theta \cap \partial^{2} \Theta$ for $\partial$ general, they all are. This implies

$$
\Theta \cap \partial \Theta \cap \partial^{2} \Theta=\bigcup_{i=1}^{6}\left(x_{i}+y_{i}+C\right),
$$

which is not possible since a general $\Theta \cap \partial \Theta \cap \partial^{2} \Theta$ has at most four irreducible components by the description of $\Theta \cap \partial_{p} \Theta \cap \partial_{p}^{2} \Theta$ above.

Since $4 p$ is not contained in a plane in $\mathbf{P}^{3}$, the curves $\Gamma_{1}$ and $\tau\left(\Gamma_{1}\right)$ defined above are disjoint. Therefore, it follows from Lemma 5 that if a general $\Theta \cap \partial \Theta \cap \partial^{2} \Theta$ is nonintegral, it is of the form $\Gamma_{0} \cup \tau\left(\Gamma_{0}\right)$, where $\Gamma_{0}$ is integral, with cohomology class $\frac{1}{2}[\Theta]^{3}$, and distinct from $\tau\left(\Gamma_{0}\right)$.

\section{Proof of Theorem 1}

We keep the same assumptions and notation as before. Let $a$ be general in $A=\operatorname{Pic}^{3}(C)$. If for some nonzero $\partial^{\prime}$, the scheme $\Theta \cap \Theta_{a} \cap \partial^{\prime} \Theta \cap \partial^{\prime} \Theta_{a}$ has dimension 1 , it contains a curve $\Gamma_{a}$ that is stable by the involution $x \mapsto a-x$. When $a$ specializes to a general $[\partial]$, this involution specializes to $\tau$, and $\Gamma_{a}$ must specialize as a set to $\Gamma_{0} \cup \tau\left(\Gamma_{0}\right)$. Since this curve has the same cohomology class as the curve $\Theta \cap \Theta_{a} \cap \partial^{\prime} \Theta$, this means that the section $\partial^{\prime} \theta_{a}$ vanishes identically on the curve $\Theta \cap \Theta_{a} \cap \partial^{\prime} \Theta$ and this is absurd.

It follows that $\psi_{a}$ is finite; hence the cotangent bundle of $\Theta \cap \Theta_{a}$ is ample.

\section{REFERENCES}

[ACGH] Arbarello, E., Cornalba, M., Griffiths, P. A., Harris, J., Geometry of algebraic curves. Vol. I, Grundlehren der Mathematischen Wissenschaften 267, Springer-Verlag, New York, 1985. MR770932 (86h:14019)

[D] Debarre, O., Varieties with ample cotangent bundle, Comp. Math. 141 (2005), 14451459. MR2188444 (2006j:14063)

[De] Demailly, J.-P., Algebraic criteria for Kobayashi hyperbolic projective varieties and jet differentials, Algebraic geometry-Santa Cruz 1995, 285-360, Proc. Sympos. Pure Math. 62, Part 2, Amer. Math. Soc., Providence, RI, 1997. MR1492539 (99b:32037)

[I] Izadi, E., Deforming curves in Jacobians to non-Jacobians. I. Curves in $C^{(2)}$, Geom. Dedicata 116 (2005), 87-109. MR2195443

[MD] Martin-Deschamps, M., Propriétés de descente des variétés à fibré cotangent ample, Ann. Inst. Fourier 34 (1984), 39-64. MR762693 (86a:14018)

[Mo] Moriwaki, A., Remarks on rational points of varieties whose cotangent bundles are generated by global sections, Math. Res. Lett. 2 (1995), 113-118. MR1312981 (96b:14021)

[N] Noguchi, J., A higher-dimensional analogue of Mordell's conjecture over function fields, Math. Ann. 258 (1981/82), 207-212. MR641826 (83d:14015)

[NS] Noguchi, J., Sunada, T., Finiteness of the family of rational and meromorphic mappings into algebraic varieties, Amer. J. Math. 104 (1982), 887-900. MR667540 (84e:14009)

IRMA - Mathématique, Université Louis Pasteur, 7 rue René Descartes, 67084 Strasbourg Cedex, France

E-mail address: debarre@math.u-strasbg.fr

Department of Mathematics, Boyd Graduate Studies Research Center, University of Georgia, Athens, Georgia 30602-7403

E-mail address: izadi@math.uga.edu 